\title{
Manutenção Aeronáutica no Brasil: distribuição geográfica e técnica
}

\section{Brazilian Aeronautical Maintenance: technical and geographical distribution}

\author{
Marcio Cardoso Machado ${ }^{1,2}$ \\ Ligia Maria Soto Urbina ${ }^{3}$ \\ Michelle Aparecida Gomes Eller ${ }^{4}$
}

\begin{abstract}
Resumo: As atividades de manutenção aeronáutica formam uma parte essencial da aeronavegabilidade continuada, tendo como objetivo, tanto na área civil quanto na militar, prover a total condição de serviço para as aeronaves. O crescimento do transporte aéreo no Brasil tem exigido uma indústria de serviços de manutenção aeronáutica capaz de apoiar tal processo expansivo, sem prejuízo para as condições de segurança de voo. Alguns dos trabalhos encontrados na revisão da literatura exploram a manutenção aeronáutica sob perspectivas que buscam melhorar a eficiência técnica do setor. Pelo exposto, o principal objetivo deste artigo é ampliar o conhecimento sobre o setor de manutenção aeronáutica no Brasil, explorando as informações providas pela ANAC, com o intuito de construir um panorama que revele a distribuição geográfica e técnica dos nichos de capacitação. Para tanto, inicia com uma exposição dos conceitos básicos da atividade de manutenção em geral e aeronáutica em particular. A seguir, é examinado o contexto regulatório brasileiro para a atividade de manutenção aeronáutica, com ênfase nos domínios de certificação. Ao final, examina-se a distribuição geográfica das empresas de manutenção aeronáutica no país, identificando a distribuição das empresas considerando-se os vários tipos de certificação técnica.
\end{abstract}

Palavras-chave: Manutenção aeronáutica; Capacitação técnica; Aeronavegabilidade; Certificação.

\begin{abstract}
The aircraft maintenance activities are an essential part of the continued airworthiness, aiming both, civilian and military areas, to provide full service condition for the aircraft. The growth of air transportation in Brazil has demanded an industry of aircraft maintenance services able to support such expansive process, without any prejudice to the flight safety conditions. Some of the studies found in the literature review, explore the aircraft maintenance from perspectives that seek to improve the technical efficiency of the sector. For these reasons, the main goal of this paper is to expand knowledge about the aircraft maintenance industry in Brazil, exploring the information provided by the ANAC in order to build a scenario that reveals the geographical and technical distribution of the existing technical training niches. For this, this study starts discussing the basic concepts of maintenance activity in general and aviation in particular. Next, we consider the Brazilian regulatory context for the aircraft maintenance activity, emphasizing the certification domain. Finally, we examine the geographic distribution of aircraft maintenance companies in the country, identifying the companies' distribution considering the various types of technical certification.
\end{abstract}

Keywords: Aircraft maintenance; Technical training; Airworthiness; Certification.

\section{Introdução}

As atividades de manutenção aeronáutica formam uma parte essencial da aeronavegabilidade continuada, tendo como objetivo, tanto na área civil quanto na militar, prover a total condição de serviço para as

aeronaves, no momento que um operador solicitar, com a qualidade esperada e com o mínimo custo (Knotts, 1999). Este serviço é de suma importância para apoiar o transporte aéreo em países como o

\footnotetext{
${ }^{1}$ Programa de Pós-graduação em Administração, Universidade Paulista - UNIP, Rua Dr. Bacelar, 1212, Vila Clementino, CEP 04026-002, São Paulo, SP, Brasil, e-mail: marciocmachado@uol.com.br

${ }^{2}$ Faculdade de Economia, Administração e Atuárias - FEA, Pontifícia Universidade Católica de São Paulo - PUC-SP, Rua Monte Alegre, 984, Perdizes, CEP 05014-901, São Paulo, SP, Brasil, e-mail: mcmachado@ pucsp.br

${ }^{3}$ Pós-graduação em Engenharia Aeronáutica e Mecânica - PG/EAM, Instituto Tecnológico de Aeronáutica - ITA, Praça Marechal Eduardo Gomes, 50, Vila das Acácias, CEP 12228-900, São José dos Campos, SP, Brasil, e-mail: ligia@ita.br

${ }^{4}$ Empresa Brasileira de Aeronáutica - Embraer, São José dos Campos, SP, Brasil, e-mail: maraujo@ita.br
}

Recebido em Dez. 20, 2012 - Aceito em Out. 24, 2014

Suporte financeiro: PNPD/CAPES, Programa Nacional de Pós-Doutorado da CAPES. 
Brasil, que se caracterizam por possuir um amplo território, com um forte trafego aéreo civil e militar que interliga as varias regiões do país. De fato, o mercado doméstico brasileiro de transporte aéreo é o maior mercado latino-americano (Araújo et al., 2007) e tem crescido à taxa de $10 \%$ entre 2003 e 2008, alcançando mais de 50 milhões de viagens por ano (Mckinsey \& Company, 2010). De acordo com esse estudo da McKinsey, é provável que este setor continue crescendo a taxas bastante relevantes, devido à expectativa de crescimento da renda e, portanto, da demanda de transporte aéreo por parte de classes e regiões mais pobres, no Brasil. Os dados comparativos do período de 2003 até de 2012, publicado pela ANAC (2012) (Agência Nacional de Aviação Civil), mostram uma taxa de crescimento da demanda do transporte aéreo, assim divididos: $205 \%$ para o transporte de passageiros e 49,76\% para o transporte de carga.

Esse crescimento do transporte aéreo requer uma indústria de serviços de manutenção aeronáutica capaz de apoiar tal processo expansivo, sem prejuízo para as condições de segurança de voo e para a aeronavegabilidade continuada. Portanto, espera-se que o setor de manutenção brasileiro receba fortes investimentos nos próximos anos, gerando retornos importantes para a economia brasileira. Nesse sentido, considera-se que a expansão, com qualidade do setor de manutenção, contribuirá para o desenvolvimento econômico do Brasil através: do aumento da participação de pequenas e médias empresas, em decorrência da existência de pequenas barreiras tecnológicas e de capital; da ampliação das oportunidades de emprego técnico e tecnológico; da disseminação geográfica da capacitação em serviços de manutenção, com a entrada destas atividades em regiões aeroportuárias com menores níveis de trafego aéreo; do estímulo ao desenvolvimento de uma indústria local de peças de reposição aeronáutica.

Alguns dos trabalhos encontrados na revisão da literatura exploram a manutenção aeronáutica sob outras perspectivas que buscam melhorar a eficiência do setor. Como exemplo, Nascimento (2006) e Rodrigues et al. (2010) estudam a perspectiva dos custos, enquanto Ando \& Costa (2004) e Papakostas et al. (2010) concentram esforços na seleção de estratégias de manutenção. Por outro lado, Vilela et al. (2010) analisam a relação da manutenção com acidentes aeronáuticos e recomendações de segurança operacional. Outros autores, ainda, focalizam assuntos clássicos da literatura de manutenção como análise dos sistemas para diagnóstico de falhas (Silva et al., 2005); da confiabilidade e sua relação com o controle de custos (Mata et al., 1998); do planejamento da manutenção (Samaranayake, 2006; Samaranayake et al., 2007). Contudo, não foi possível identificar pesquisas relacionadas com a distribuição geográfica e também com relação às capacitações técnicas, que seriam úteis no suporte à expansão do transporte aéreo.

Nesse contexto, este trabalho tem como objetivo ampliar o conhecimento sobre o setor de manutenção aeronáutica no Brasil, explorando as informações providas pela ANAC (2010), com o intuito de construir um panorama que revele a distribuição geográfica e técnica dos nichos de capacitação técnica. E, para tanto, construiu-se a seguinte questão de pesquisa: como se distribuem, geográfica e tecnicamente, as empresas de manutenção aeronáutica brasileiras?

Mais especificamente, este trabalho se inicia com uma exposição dos conceitos básicos da atividade de manutenção em geral e aeronáutica em particular. A seguir, examina-se o contexto regulatório brasileiro para a atividade de manutenção aeronáutica, com ênfase nos domínios de certificação. Logo é realizada uma pesquisa exploratória a partir de dados primários com o intuito de detectar concentrações de natureza locacional e técnica. Com esse foco, examina-se a distribuição geográfica das empresas de manutenção aeronáutica no país. Também, o estudo permite identificar a distribuição das empresas, considerando-se os vários tipos de certificação técnica.

\section{A atividade de manutenção}

Para alcançar desempenho de classe mundial, as empresas estão empenhando esforços no sentido de aumentar a qualidade e a produtividade, e reduzir custos (Mishra et al., 2006). Para determinadas empresas, parte desses esforços deve incluir uma análise das atividades da função manutenção. Uma manutenção efetiva é fundamental para muitas operações. É a partir dela que podemos estender o ciclo de vida do produto, melhorar a disponibilidade dos equipamentos e mantê-los em bom estado. Por outro lado, o descuido com a manutenção pode produzir falhas mais frequentes, subutilização dos equipamentos e o consequente atraso nos calendários de produção. Segundo Waeyenbergh \& Pintelon (2002), as empresas possuem um grande número de sistemas que interagem entre si para alcançar os objetivos organizacionais. Na maioria das empresas, manutenção é uma atividade de suporte à competência essencial da organização, que corresponde à razão de sua sobrevivência e é central na sua estratégia de diferenciação perante concorrentes e clientes (Fleury \& Fleury, 2003, 2004; Prahalad \& Hamel, 1990). Como atividade de apoio, a manutenção tem como objetivo manter operacionais os equipamentos que estão sob sua responsabilidade. Por outro lado, segundo Martin (1997), existem outras empresas para as quais as competências corporativas de nível mais elevado centram-se na atividade de prestação de serviços de manutenção. Reforça-se ainda que a manutenção, em seu processo evolutivo, tem assumido cada vez mais um papel de destaque, não apenas no setor industrial, 
mas também nos mais variados tipos de serviços, como transportes (Alves \& Falsarella, 2009).

Para Pun et al. (2002) e Pintelon et al. (2006), a manutenção, como uma função estratégica de suporte aos negócios, desempenha um importante papel em apoiar diferentes técnicas de gerenciamento da produção emergentes ou já consolidadas (ex.: produção enxuta, just-in-time e produção seis sigma). A eficácia no gerenciamento da manutenção depende do desdobramento adequado dos recursos, sejam estespeças de reposição, ferramentas, equipamentos ou mão de obra. Esta característica impõe um caráter estratégico nas atividades de manutenção. Conforme Tsang (1998), considerar a manutenção apenas como um elemento tático dentro das empresas é uma miopia, ela tem também uma dimensão estratégica com desdobramentos para o projeto de instalações e dos programas de manutenção, atualização do conhecimento e habilidades da força de trabalho e o desdobramento da carga de trabalho e ferramentas para execução das diferentes atividades de manutenção. $\mathrm{O}$ gerenciamento da manutenção torna-se, portanto, um elemento importante a ser estudado de forma científica, e é o que tem ocorrido, conforme o levantamento realizado no trabalho de Garg \& Deshmukh (2006).

Para Sherwin (2000), os sistemas de manutenção devem ser moldados de acordo com a natureza do trabalho que será gerenciado. Ou seja, na indústria siderurgia serão estabelecidos procedimentos de gerenciamento da manutenção que irão diferir daqueles utilizados, por exemplo, na indústria aeronáutica.

$\mathrm{Na}$ indústria aeronáutica, podemos identificar claramente empresas que têm a manutenção como função organizacional e empresas que têm a manutenção como atividade-fim. Empresas de transporte aéreo de carga ou de passageiros, normalmente, têm em sua estrutura organizacional um departamento responsável pela manutenção de seus equipamentos (aeronaves). Contudo, existem empresas que prestam serviços de manutenção aeronáutica nos equipamentos que constituem os diferentes sistemas das aeronaves. Por exemplo, uma companhia aérea, ao realizar a manutenção em um de seus equipamentos, identifica a necessidade de troca de um motor; esse motor, muito provavelmente, será enviado a uma empresa especializada que fará o devido reparo.

Assim como a manutenção aeronáutica exige requisitos que são inerentes às suas operações, podemos estabelecer ainda diferenças entre a manutenção aeronáutica militar e civil. Segundo Chockie \& Bjorkelo (1992), as filosofias que governam as operações e manutenção de uma planta diferem quando comparamos organizações comerciais e militares. Os programas de manutenção militares não sofrem as mesmas restrições que as organizações comerciais. Como exemplo, os autores citam que as operações continuadas de uma aeronave militar são orientadas por requisitos estratégicos impostos pelo Comando da Aeronáutica e não por razões econômicas. Os requisitos impostos especificam certo número de missões e disponibilidade da frota, independentemente dos custos envolvidos.

\subsection{Manutenção aeronáutica}

A manutenção aeronáutica pode ser dividida em duas atividades que, apesar de estarem completamente associadas, possuem especificidades que as distinguem. A primeira atividade se refere à manutenção das aeronaves como um equipamento único, e a segunda atividade se refere à manutenção dos componentes que servirão como insumos para a primeira. Esta distinção se faz necessária, visto que as características das operações de manutenção de aeronaves seguem regras que vão além da competência técnica necessária para a execução atividades de manutenção. Um dos exemplos disso é a necessidade de um combate intenso contra a ocorrência do erro humano na execução da tarefa, pois uma aeronave, após a manutenção, não pode ser testada da mesma forma que um equipamento, o qual é colocado muitas vezes em uma bancada de testes para simulação de condições de voo. Contudo, isto não significa que o erro humano na manutenção de componentes seja tolerado, mas os princípios que norteiam o seu combate estão mais associados à qualidade do processo do que propriamente a questões de segurança de voo. Desta forma, conforme explicam Cheung et al. (2005), existe uma dificuldade de alocação de mão de obra especializada na manutenção aeronáutica.

\subsection{Tipos de manutenção}

A manutenção aeronáutica também pode ser classificada como manutenções preventivas (hard time), manutenções corretivas (condition monitoring) ou manutenções preditivas (on condition) (Knotts, 1999).

O modelo apresentado na Figura 1 é uma visão simplificada da relação entre a manutenção aeronáutica com as suas três classificações e a manutenção dos componentes (itens) aeronáuticos.

a) manutenção preventiva - Para Tu et al. (2001), a manutenção preventiva é a prática de substituir componentes ou subsistemas antes que eles falhem, normalmente com frequencia predeterminada (hard time) ou em virtude de inspeção e teste. O objetivo é manter a operação contínua do sistema, nesse caso a aeronave;

b) manutenção corretiva - Para Moayed \& Shell (2009), é aquela que ocorre após a identificação e diagnóstico de um problema (condition monitoring). Durante este diagnóstico, os técnicos de manutenção têm que identificar as partes que falharam e fazer as respectivas ações de reparo; 
c) manutenção preditiva - leva em conta o contínuo acompanhamento dos limites de operação de um dado componente ou subsistema (on-condition). Verificada qualquer tendência para a ocorrência de uma falha funcional do componente ou subsistema, este deve ser removido para manutenção. Alguns mecanismos para a execução da manutenção preditiva são o PdM (Product Data Management)/PHM (Produc History Management).

\subsection{Manutenção aeronáutica no Brasil}

Esta parte do trabalho visa descrever sucintamente o setor de manutenção aeronáutica no Brasil.

\subsubsection{Contexto regulatório da manutenção aeronáutica brasileira}

A Agência Nacional de Aviação Civil - ANAC tem como missão promover a segurança e a excelência do sistema de aviação civil, de forma a contribuir para o desenvolvimento do país e o bem-estar da sociedade brasileira. Portanto, é atribuição dela estabelecer e fiscalizar o cumprimento do marco regulatório que rege as atividades das empresas de manutenção.

No que se refere às atividades das oficinas de manutenção aeronáutica, a ANAC (2010) classifica as empresas de acordo com o tipo de serviços que elas são capazes de executar. Assim, elas podem ser oficinas de manutenção de aeronaves, de células, de motores, de hélices, de rotores, de equipamentos e de partes dos referidos conjuntos.

Para essa divisão de categorias de manutenção, a ANAC também estabeleceu categorias, classes e limitações para a atividade de manutenção. Desta forma, qualquer empresa que deseja ser classificada como uma oficina de manutenção aeronáutica deverá apresentar uma solicitação de homologação à ANAC, especificando em qual aeronave, motor, hélice, rotor, equipamento ou parte irá executar o serviço de manutenção. Com base no Regulamento Brasileiro de Aviação Civil (RBAC) 145 (Brasil, 2014), são avaliadas as qualificações técnica e organizacional da empresa, que, quando confirmadas, tem como contrapartida a emissão de um Certificado de Organização de Manutenção - COM (Brasil, 2014).

\subsubsection{Domínios técnicos de certificação}

Os Certificados de Organização de Manutenção emitidos pela ANAC (Brasil, 2014) referem-se às empresas de manutenção aeronáutica e baseiam-se nas categorias e classes constantes do Quadro 1.

As oficinas de manutenção nacionais podem ser certificadas, de acordo com seu domínio técnico de certificação, em qualquer uma das classes constantes no Quadro 1. Ou também podem obter certificação em mais de uma classe de uma mesma categoria ou de categorias diferentes. Por exemplo, segundo dados da ANAC (2010), a oficina VRG Montevideu

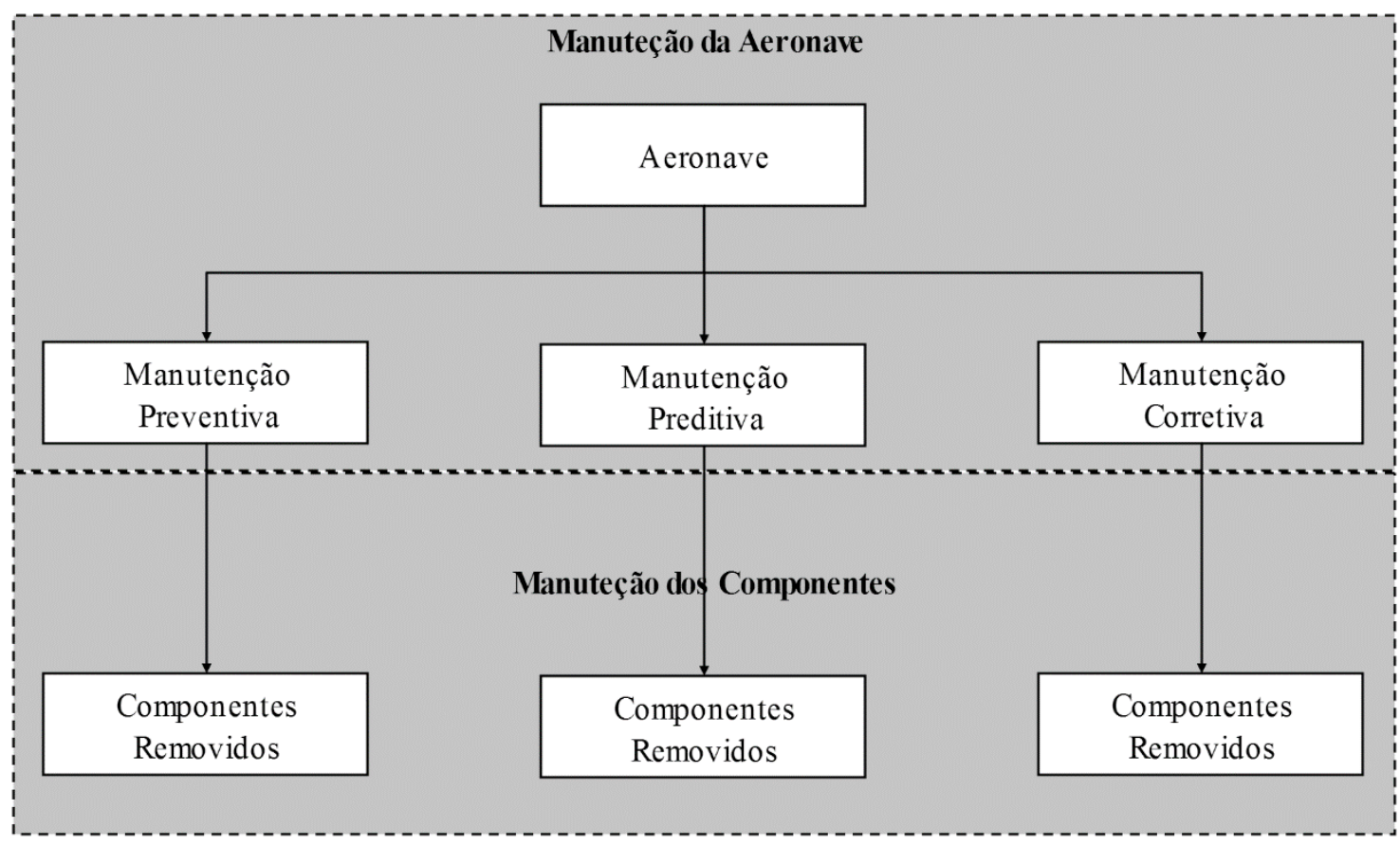

Figura 1. Modelo de manutenção aeronáutica. 
Quadro 1. Categorias e classes de empresas de manutenção aeronáutica.

\begin{tabular}{|c|c|}
\hline Categoria & Classe \\
\hline $\begin{array}{l}\text { Célula - Manutenção, } \\
\text { modificações e reparos } \\
\text { em células }\end{array}$ & $\begin{array}{l}\text { Classe } 1 \text { - Aeronaves fabricadas com material composto, com peso máximo de decolagem } \\
\text { aprovado até } 12500 \mathrm{lbf}(5670 \mathrm{kgf}) \text { no caso de aviões ou } 6018 \mathrm{lbf} \text { ( } 2730 \mathrm{kgf}) \text { no caso de } \\
\text { helicópteros. } \\
\text { Classe } 2 \text { - Aeronaves fabricadas com material composto, com peso máximo de decolagem } \\
\text { aprovado acima de } 12500 \mathrm{lbf} \text { ( } 5670 \mathrm{kgf}) \text { no caso de aviões ou } 6018 \mathrm{lbf} \text { ( } 2730 \mathrm{kgf}) \text { no caso } \\
\text { de helicópteros. } \\
\text { Classe } 3 \text { - Aeronaves fabricadas em estrutura metálica, com peso máximo de decolagem } \\
\text { aprovado até } 12500 \mathrm{lbf}(5670 \mathrm{kgf}) \text { no caso de aviões ou } 6018 \mathrm{lbf} \text { ( } 2730 \mathrm{kgf}) \text { no caso de } \\
\text { helicópteros. } \\
\text { Classe } 4 \text { - Aeronaves fabricadas em estrutura metálica, com peso máximo de decolagem } \\
\text { aprovado acima de } 12500 \mathrm{lbf} \text { ( } 5670 \mathrm{kgf}) \text { no caso de aviões ou } 6018 \text { lbf ( } 2730 \mathrm{kgf}) \text { no caso } \\
\text { de helicópteros. }\end{array}$ \\
\hline $\begin{array}{l}\text { Motor - Manutenção, } \\
\text { modificações e } \\
\text { reparos em motores de } \\
\text { aeronaves }\end{array}$ & $\begin{array}{l}\text { Classe } 1 \text { - Motores convencionais com até } 400 \mathrm{hp}(298 \mathrm{~kW}) . \\
\text { Classe } 2 \text { - Motores convencionais com mais de } 400 \mathrm{hp}(298 \mathrm{~kW}) . \\
\text { Classe } 3 \text { - Motores à turbina. }\end{array}$ \\
\hline $\begin{array}{l}\text { Hélice - Manutenção, } \\
\text { modificações e reparos } \\
\text { em hélices e rotores de } \\
\text { aeronaves }\end{array}$ & $\begin{array}{l}\text { Classe } 1 \text { - Hélices de madeira, metal ou material composto, de passo fixo ou ajustável no } \\
\text { solo. } \\
\text { Classe } 2 \text { - Outras hélices. }\end{array}$ \\
\hline $\begin{array}{l}\text { Rádio - Manutenção } \\
\text { e reparos em } \\
\text { equipamentos de } \\
\text { aeronaves }\end{array}$ & $\begin{array}{l}\text { Classe } 1 \text { - Equipamentos de comunicação. Equipamentos de radiotransmissão e/ou } \\
\text { recepção utilizados em uma aeronave para enviar ou receber comunicações em voo, } \\
\text { independente da frequência da portadora ou tipo de modulação utilizado. Esta classe } \\
\text { inclui sistemas auxiliares e sistemas de interfones da aeronave, sistemas de amplificação, } \\
\text { dispositivos de sinalização da tripulação, elétrico ou eletrônico e equipamentos similares. } \\
\text { Esta classe não inclui os equipamentos usados para navegação ou auxílio à navegação } \\
\text { da aeronave, equipamento usado para medir altitude ou separação do solo, outros } \\
\text { equipamentos de medida operados pelos princípios de rádio ou radar, ou instrumentos } \\
\text { mecânicos, elétricos, giroscópicos ou eletrônicos, que são parte de um equipamento de } \\
\text { radiocomunicação. } \\
\text { Classe } 2 \text { - Equipamentos de navegação. Sistemas de rádio utilizados em uma aeronave em } \\
\text { navegação de rota ou de aproximação. Não inclui equipamentos operados pelos princípios } \\
\text { de radar ou de pulsos de radiofrequência, ou equipamento utilizado para medir altitude ou } \\
\text { separação do solo. } \\
\text { Classe } 3 \text { - Equipamentos de radar. Sistemas eletrônicos da aeronave que operam pelos } \\
\text { princípios de radar ou de pulsos de radiofrequência. }\end{array}$ \\
\hline Instrumento & $\begin{array}{l}\text { Classe 1: Mecânico. Instrumentos com diafragma, tubo Bourdon, aneroide, óptico, ou } \\
\text { instrumento acionado mecanicamente por força centrífuga, usado na aeronave ou para } \\
\text { operar a aeronave, incluindo tacômetros, indicadores de velocidade, manômetros de } \\
\text { pressão, bússolas, altímetros ou instrumentos mecânicos similares. } \\
\text { Classe 2: Elétrico. Sistemas e instrumentos de indicação, elétricos e autossíncronos, } \\
\text { incluindo instrumentos de indicação remota, instrumentos de indicação de temperatura de } \\
\text { cabeça de cilindro, ou instrumentos elétricos similares. } \\
\text { Classe 3: Giroscópios. Instrumentos ou sistemas que utilizam princípio giroscópico e } \\
\text { movidos a pressão de ar ou energia elétrica, incluindo unidades de controle de piloto } \\
\text { automático, indicadores de turn-bank, giros direcionais e suas partes, bússolas flux gate e } \\
\text { gyrosyn. } \\
\text { Classe 4: Eletrônico. Instrumentos cuja operação depende de válvulas eletrônicas, } \\
\text { transistores ou dispositivos similares, incluindo medidores de quantidade por capacitância, } \\
\text { sistemas amplificadores e analisadores de motor. }\end{array}$ \\
\hline
\end{tabular}

Fonte: Brasil (2014). 
Quadro 1. Continuação...

\begin{tabular}{|l|l|}
\hline \multicolumn{1}{|c|}{ Categoria } & \multicolumn{1}{c|}{ Classe } \\
\hline \multirow{5}{*}{ Acessório } & $\begin{array}{l}\text { Classe 1: Acessórios mecânicos que dependem de atrito, hidráulica, acoplamento } \\
\text { mecânico, ou pressão pneumática para sua operação, incluindo freios de roda de aeronave, } \\
\text { bombas acionadas mecanicamente, carburadores, conjunto de rodas de aeronave, } \\
\text { amortecedores, unidades servo hidráulicas e equipamentos de emergência. } \\
\text { Classe 2: Acessórios elétricos que dependem de energia elétrica para sua operação, } \\
\text { geradores, motores de partida, reguladores de voltagem, motores elétricos, bombas de } \\
\text { combustível acionadas eletricamente, magnetos, ou acessórios elétricos similares. } \\
\text { Classe 3: Acessórios eletrônicos que dependem do uso de válvulas eletrônicas, transistor } \\
\text { ou dispositivos similares, incluindo sistemas de entretenimento em voo, controles de } \\
\text { superalimentador, de temperatura e ar condicionado ou controles eletrônicos similares. }\end{array}$ \\
\hline $\begin{array}{l}\text { Classe Única: Atividades específicas de execução de manutenção que a ANAC julgar } \\
\text { procedente caso não esteja sob outras certificações de organização de manutenção, por } \\
\text { tipo de serviço (ex.: ensaios não destrutivos, serviços de soldagem, pintura, pesagem de } \\
\text { aeronaves, trabalhos em revestimentos de tela, serviços especializados em pás de rotores, } \\
\text { especializados } \\
\text { análises de vibração e balanceamento dinâmico, análises de performance, serviços de } \\
\text { tapeçaria e interiores, inspeções e testes do sistema anemométrico, inspeção boroscópica, } \\
\text { lavagem de compressores de motores à reação, banhos galvânicos, shot peening, limpeza } \\
\text { por jateamento abrasivo, inspeção por ataque ácido, inspeções/ensaios de vasos de } \\
\text { pressão). }\end{array}$ \\
\hline
\end{tabular}

Fonte: Brasil (2014).

(VRG Linhas Aéreas S.A.) é certificada para realizar serviços na Categoria Célula, Classe 4, ou seja, esta oficina pode realizar manutenção em aeronaves fabricadas em estrutura metálica, com peso máximo de decolagem aprovado acima de $12500 \mathrm{lbf}$ (5670 kgf) no caso de aviões ou 6018 lbf (2730 kgf) no caso de helicópteros. Outra empresa, a Rolls-Royce São Bernardo do Campo (Rolls-Royce Brasil Ltda.), pode realizar serviços de manutenção nas seguintes categorias: Motor Classe 3 e na Categoria Acessórios Classe 1, que respectivamente incluem serviços de: manutenção, modificações e reparos em motores de aeronaves à turbina e manutenção e reparos em acessórios mecânicos de aeronaves, por modelo de acessório.

O escopo das certificações vai depender do interesse e da capacitação técnica das oficinas para realização dos serviços que pretendem oferecer no mercado.

O Quadro 1 detalha a competência técnica em cada Categoria e Classe de certificação:

Para efeito deste trabalho, as categorias Rádio, Instrumentos e Acessórios foram agrupadas em uma só categoria, que a partir de agora serão tratadas como categoria Equipamentos. Essa consolidação se dá ao fato de que os dados iniciais coletados nessa pesquisa, e obtidos com a ANAC (2010), só estavam disponíveis desta forma.

\section{Método de pesquisa}

Após definir o problema e elaborar a questão de pesquisa, iniciou-se a parte exploratória que constou inicialmente da elaboração de um referencial teórico, cujos principais objetivos foram: encontrar desenvolvimentos recentes sobre o tema, identificar os principais autores e periódicos da área e destacar a necessidade da pesquisa.

Feita a aproximação com a literatura, e verificada a consistência da questão de pesquisa, foi elaborado um projeto do estudo. Segundo Quivy \& Van Campenhoudt (1998), um projeto de estudo é o caminho para alcançar os objetivos e responder as questões de pesquisa. Muitas vezes, elaborar projeto pode ser complicado em razão da disponibilidade de variados métodos, técnicas, procedimentos, protocolos e planos de amostragem. Por exemplo, escolher entre estudo de caso, survey, experimento ou pesquisa-ação (Voss et al., 2002).

O projeto de estudo desta pesquisa pode ser classificado com a utilização das seguintes perspectivas: trata-se de uma pesquisa qualitativa com uma abordagem exploratória e também descritiva realizada a partir dos dados documentais primários publicados no site da ANAC (2010). Para Cooper \& Emory (1995), dados governamentais são boas fontes primárias de informação em muitas áreas, porém, por vezes, seu acesso é restrito, inviabilizando a pesquisa. Ainda segundo os autores, os dados online, via Internet, podem ser caros por necessitarem pagamento de assinaturas, taxas para impressão, etc. Contudo, no caso específico deste artigo, os dados governamentais da ANAC estavam gratuitamente disponíveis na Internet, fato que está se tornando cada vez mais comum com dados obtidos via Internet (Bryman \& Bell, 2007).

A extração dos dados do site da ANAC (2010) seguiu as seguintes etapas propostas por Cooper \& Emory (1995): seleção os dados que seriam necessários à pesquisa, sendo as informações desnecessárias 
descartadas, e transferência dos dados para uma planilha eletrônica. Mais especificamente, foram coletadas as seguintes informações: o nome da empresa, tipos de certificação obtidos, localização geográfica (estado) e contato (telefone e e-mail).

A análise dos dados constou inicialmente da quantificação do número de oficinas por localização e por tipo de certificação. Em seguida foi feita uma correlação entre número de oficinas e quantidade de aeronaves por estado. Por fim, fez-se uma análise da concentração técnica, levando em conta os tipos de certificação obtidos pelas oficinas. Por fim, foram apresentados os resultados obtidos.

Não foi possível a descrição completa dos procedimentos utilizados nesta pesquisa, em virtude da limitação de espaço. Contudo, a Figura 2 apresenta a estrutura metodológica utilizada.

Basicamente, o intuito é construir uma visão abrangente sobre o setor, que destaque principalmente as suas características de natureza técnica e locacional.

\section{Dados para a pesquisa}

Com relação aos dados, deve-se salientar que as informações sobre as oficinas analisadas neste trabalho foram coletadas uma a uma, em função da indisponibilidade de uma lista de oficinas de manutenção aeronáutica no site da ANAC (2010). Assim, os dados foram consolidados em uma planilha onde cada oficina foi discriminada segundo sua certificação e localização no espaço geográfico brasileiro, considerando-se as regiões Norte, Sul, Sudeste, Centro-Oeste e Nordeste.

A pesquisa contou com dados de 596 oficinas listadas no site da ANAC (2010). Do total dessas oficinas, a

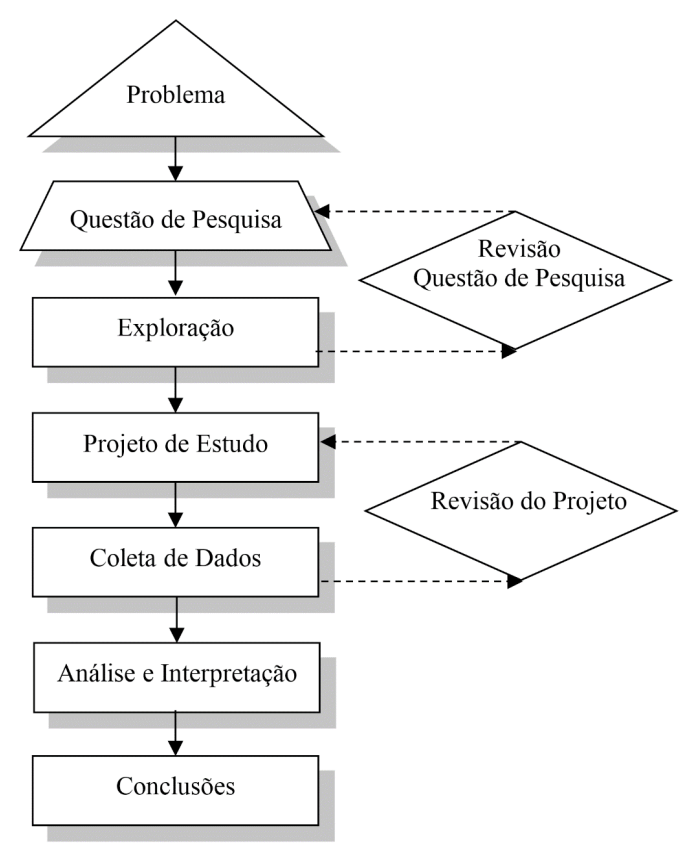

Figura 2. Estrutura do método de pesquisa. maioria (487) está situada em território nacional, e um pequeno número (109) são oficinas localizadas fora do território nacional, mas que possuem certificação ANAC para realização de serviços aeronáuticos (Tabela 1).

Com relação às certificações, pode-se observar que 70\% das oficinas possuem algum tipo de certificado Categoria "Célula", ou seja, realizam serviços de manutenção, modificações e reparos em células. Pode-se entender como célula da aeronave a sua estrutura metálica ou mista (madeira, alumínio e/ou material composto).

O somatório do percentual de oficinas com certificação nas respectivas categorias não totalizar exatamente $100 \%$ se deve ao fato de uma grande parte das oficinas (468) possuir diferentes certificações em diferentes Categorias.

Existe um pequeno número de oficinas (8) que possuem o Certificado de Aceitação de Empresa de Manutenção - CAEM, que é um certificado emitido pela ANAC para fins de reconhecimento de capacitação técnica de oficinas de manutenção fora do Brasil. Atualmente, esses certificados têm sido emitidos para empresas canadenses, em razão de um acordo bilateral entre a ANAC e o Transport Canada Civil Aviation - TCCA.

No que diz respeito às regiões, observa-se uma concentração de oficinas na região Sudeste, o que não representa uma informação inesperada para a pesquisa, visto que existe também uma concentração da frota de aeronaves nessa região. Segundo análise realizada, verificou-se que das 12178 aeronaves registradas no Brasil, 5443, ou seja, 44,7\%, estão na região sudeste do país (ANAC, 2010).

Outro aspecto observado é que $68 \%$ das oficinas de manutenção possuem até três certificações inclusive, e $32 \%$ quatro ou mais certificações. Essa informação reforça a ideia de uma especialização das empresas, ou seja, que mesmo possuindo varias certificações, estas certificações estão relacionadas com uma mesma família de padrão. Por exemplo, a empresa que possui a certificação Categoria Hélice Classe 1 provavelmente possuirá também outra certificação Categoria Hélice Classe 2.

\section{Distribuiç̧ão geográfica e concentração técnica dos nichos de capacitação}

\subsection{Distribuição geográfica das empresas}

Na Tabela 2 estão descritos os dados referentes à quantidade de aeronaves e de oficinas de manutenção, por estados. A ordem utilizada para a construção da Tabela 2 foi da maior para a menor quantidade de aeronaves por estado.

A Figura 3 revela a correlação entre o número de aeronaves por estado e a população de empresas de manutenção. São Paulo, por exemplo, é o estado que concentra o maior número de aeronaves (3.641) 
Tabela 1. Dados da amostra.

\begin{tabular}{cccc}
\hline & Categoria & $\begin{array}{c}\text { Quantidade } \\
\text { de oficinas }\end{array}$ & \% de Oficinas \\
\hline Certificações & Célula & 418 & 70 \\
& Motor & 342 & 57 \\
& Hélice & 71 & 12 \\
& Equipamentos: Rádio, Instrumentos e Acessórios & 356 & 58 \\
& Serviços Especializados & 260 & 44 \\
Região & CAEM & 8 & 1 \\
& Norte & 46 & 8 \\
& Nordeste & 35 & 6 \\
& Sudeste & 260 & 44 \\
Quantidade de & Centro-oeste & 73 & 12 \\
Certificações & Sul & 73 & 12 \\
& Exterior & 109 & 18 \\
\end{tabular}

Tabela 2. Quantidade de aeronaves e oficinas por estado.

\begin{tabular}{cccccc}
\hline Estado & Qtde Aeronaves & Qtde Oficinas & Estado & Qtde Aeronaves & Qtde Oficinas \\
\hline SP & 3641 & 162 & RR & 147 & 2 \\
MT & 941 & 23 & RO & 143 & 1 \\
MG & 911 & 34 & MA & 142 & 3 \\
RS & 891 & 41 & PE & 109 & 7 \\
RJ & 818 & 60 & TO & 91 & 2 \\
GO & 753 & 29 & PI & 88 & 3 \\
PR & 718 & 25 & ES & 73 & 4 \\
PA & 715 & 15 & AC & 63 & 1 \\
MS & 540 & 14 & AL & 52 & 2 \\
DF & 281 & 7 & PP & 30 & 1 \\
BA & 280 & 12 & RN & 36 & 2 \\
SC & 262 & 7 & SE & 17 & 4 \\
AM & 210 & 14 & & & 2 \\
CE & 172 & 10 & & & \\
\hline
\end{tabular}

e também o maior número de oficinas de manutenção certificadas (162).

Foi possível verificar também que $80,9 \%$ das aeronaves estão registradas nas regiões Sudeste, Sul e Centro do país, levando o percentual de oficinas nessas mesmas regiões totalizarem $84,4 \%$, excluindo as oficinas localizadas fora do Brasil.

Esta análise nos leva a confirmar o pressuposto esperado de que as oficinas de manutenção aeronáutica tendem a se aproximar geograficamente dos mercados onde o número de clientes pode ser maior. Esta confirmação nos leva a considerar mais alguns pressupostos: primeiro, pode-se entender que em parte esta correlação se deve ao fato de que o custo de movimentação desses equipamentos (aeronaves e componentes) para oficinas de manutenção fora das

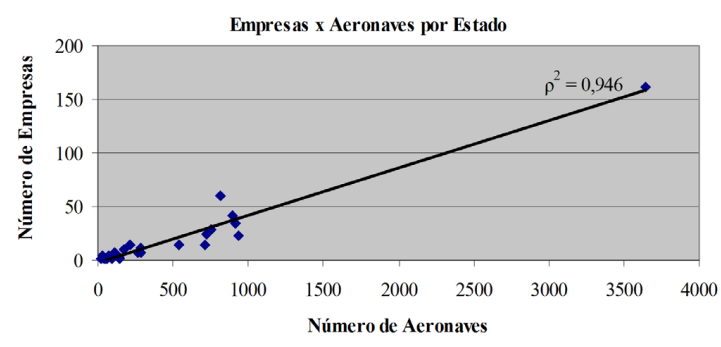

Figura 3. Número de aeronaves $\mathrm{x}$ número de oficinas por Estado.

suas bases poderia ser diminuído se estas oficinas estivessem próximas. Segundo, de que as manutenções das aeronaves são feitas pelos próprios operadores, resultando a forte correlação de proximidade geográfica. 
Como essa primeira análise utilizou o total de certificações sem considerar a distribuição das categorias, cabe verificar se há diferença significativa com relação às diferentes categorias e a quantidade de aeronaves nos estados.

\subsection{Concentração técnica}

Conforme já descrito no Quadro 1, as empresas de manutenção aeronáutica são classificadas em função do tipo de serviço que executam. Na Tabela 1 estão representados os percentuais de empresas certificadas em cada uma das categorias relativos do total de oficinas de manutenção existentes no Brasil. No gráfico da Figura 4 aparecem os percentuais de cada uma das categorias relativas ao total de certificações emitidas. Neste caso, $30 \%$ é Categoria Célula, $20 \%$ é Categoria Motor, 6\% é Categoria Hélice, 30\% é Categoria Equipamentos e $14 \%$ é Serviços Especializados.

Na Tabela 3 é possível comparar os dados a respeito das oficinas certificadas em cada categoria com relação ao total de oficinas certificadas pela ANAC, e também das certificações emitidas por categoria e o total de

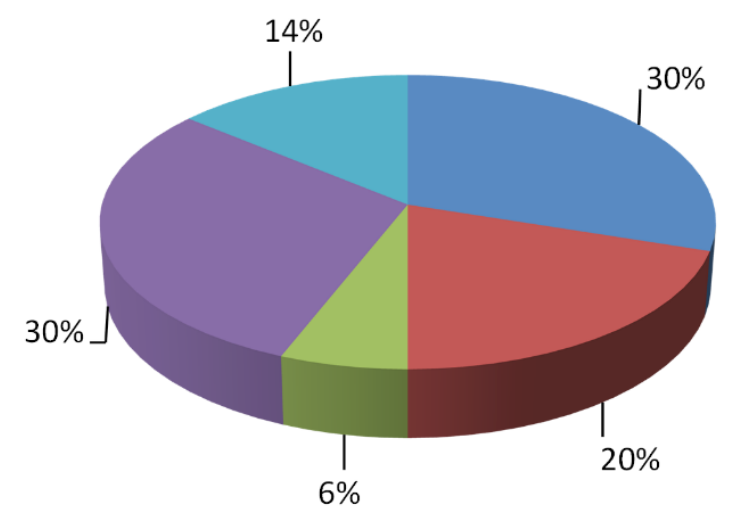

\begin{tabular}{|l|}
\hline Célula \\
Motor \\
Hélice \\
Equipamentos: Rádio, \\
Instrumentos e Acessórios \\
serviços Especializados \\
\hline
\end{tabular}

Figura 4. Percentual de certificações por categoria. certificações emitidas pela ANAC. Nesta análise foram considerados: um universo de 596 oficinas e um universo de 1.908 certificações. Ainda na Tabela 3, é possível verificar que o total de oficinas certificadas na Categoria Célula, em qualquer uma de suas classes, é de 418 oficinas, o que representa $70 \%$ do universo pesquisado. Já o número de certificados na Categoria Célula, considerando também todas as classes, corresponde a 583 certificados, ou seja, $30 \%$ do universo. A análise da Tabela 3 ratifica que as empresas são certificadas em mais de uma categoria, conforme já mencionado no item 4 deste trabalho.

A maior parte das oficinas (70\%) tem certificação na Categoria Célula, porém observa-se que esta certificação não é predominante na média, pois tal categoria representa $30 \%$ das certificações totais. Isto nos leva a inferir que as empresas que detêm certificações na Categoria Célula, em qualquer uma das suas classes, não terão necessariamente outras certificações dentro da mesma família de tal Categoria.

Nessa linha de raciocínio, pode-se arguir que as muitas empresas com certificações na Categoria Célula também possuem certificações em outras categorias, o que se manifesta em uma distribuição menos concentrada das certificações, onde as Categorias Motor e Equipamentos representam cerca de 50\% do total de certificados emitidos, conforme apresentado na coluna Certificados/Total de certificados da Tabela 3.

\subsection{Conclusões e recomendações para trabalhos futuros}

Os dados aqui apresentados representam um estudo preliminar da indústria de manutenção aeronáutica brasileira. Consequentemente, trata-se de uma abordagem exploratória e descritiva sem a pretensão de generalização para a manutenção aeronáutica como um todo, o que inclui oficinas também fora do Brasil. Contudo alguns pontos podem ser discutidos a partir da análise feita neste trabalho e, na medida em que esses pontos forem sendo apresentados, serão também colocados questionamentos para futuras pesquisas.

No que diz respeito aos tipos de certificações, verificou-se que a quantidade de empresas certificadas na Categoria Célula (70\%) supera significativamente

Tabela 3. Categorias por quantidade de oficinas e por quantidade de certificações.

\begin{tabular}{lccccc}
\hline & Categoria & $\begin{array}{c}\text { Oficinas } \\
\text { Certificadas }\end{array}$ & $\begin{array}{c}\text { Oficinas Certificadas/ } \\
\text { Total de Oficinas }\end{array}$ & Certificados & $\begin{array}{c}\text { Certificados/ } \\
\text { Total de certificados }\end{array}$ \\
\hline Certificações & Célula & 418 & $70 \%$ & 583 & $30 \%$ \\
& Motor & 342 & $57 \%$ & 388 & $20 \%$ \\
& Hélice & 71 & $12 \%$ & 105 & $6 \%$ \\
& Equipamentos & 356 & $58 \%$ & 564 & $30 \%$ \\
Universo & Serviços Esp. & 260 & $44 \%$ & 260 & $14 \%$ \\
\hline
\end{tabular}


as empresas certificadas na Categoria Motor (57\%) e na Categoria Serviços Especializados (58\%). Em função disso, surgem algumas questões: considerando que a especialização e a capacitação técnica para execução dos serviços nas Categorias Motor e Serviços Especializados são significativamente maiores do que aqueles exigidos para manutenção nas células de aeronaves, pode-se entender que a nossa indústria de manutenção aeronáutica está defasada tecnologicamente em relação a outras indústrias? O volume de serviço na Categoria Célula supera aqueles demandados pelas Categorias Motor e Serviços Especializados?

Foi possível observar também que existe um grande número de oficinas que detêm certificação para mais de uma classe na mesma categoria ou em categorias diferentes. Portanto, questiona-se: isto é uma prática usual no cenário de manutenção aeronáutica internacional? Até que ponto a certificação casada de diferentes categorias pode afetar o desenvolvimento das competências técnicas inerentes a cada categoria?

Este trabalho, portanto, procurou trazer para a discussão científica o tema da manutenção aeronáutica e, a partir dos dados coletados, avaliar as empresas de manutenção aeronáutica do ponto de vista da distribuição geográfica e também estudar as informações acerca do tipo de certificações que cada empresa possui, ou seja, a capacitação técnica.

A partir deste trabalho pôde-se identificar a necessidade de pesquisas para obter informações a respeito de volumes de serviço executado por essas empresas e melhorar a visão panorâmica da situação de empresas que prestam serviço de manutenção de aeronaves e de manutenção de componentes. A análise dessa dicotomia pode trazer informações relevantes sobre a proporção entre o serviço de manutenção de aeronaves e de manutenção de componentes, assim como da localização de empresas de manutenção de componentes, que a princípio não precisam estar próximas aos mercados consumidores. E da localização dos serviços de manutenção de aeronaves que, em hipótese, dependem em muito do volume de aeronaves que chega à região, o que no trabalho se manifestou como uma forte correlação entre o número de aeronaves e o número de empresas, e que talvez diminuiria se fosse considerado apenas o caso das empresas de manutenção de componentes. É preciso investigar ainda se existem outros fatores que impulsionam a aglomeração das empresas de manutenção na região Sudeste do país.

Por fim, a partir desta pesquisa, descortinou-se a necessidade de recomendar que trabalhos futuros realizem uma verificação das práticas operacionais e de gestão desenvolvidas nesse setor, também examinem como os atores que compõem este cenário estão se capacitando, tanto em termos organizacionais como em termos técnicos, haja vista a grande importância o tema.

\section{Referências}

Agência Nacional de Aviação Civil - ANAC (2010). Empresas de manutenção aeronáutica certificadas. Recuperado em 19 de maio de 2010, de http://www. anac.gov.br

Agência Nacional de Aviação Civil - ANAC (2012). Anuário do transporte aéreo: dados estatísticos $e$ econômicos de 2012. Brasília: ANAC. 188 p.

Alves, R. P., \& Falsarella, O. M. (2009). Modelo conceitual de inteligência organizacional aplicada à função manutenção. Gestão \& Produção, 16(2), 313-324.

Ando, J. K., \& Costa, H. G. (2004). Seleção de estratégias de manutenção em operadoras de táxi aéreo "offshore": modelagem pelo ELECTRE I. In Anais do XXIV Encontro Nacional de Engenharia de Produção (pp. 1-8). Florianópolis: ABEPRO.

Araujo, A. H., Jr., Avellar, J. V. G., Marins, F. S. \& Milioni, A. Z. (2007). A eficiência operacional do transporte aéreo brasileiro. Engenharia, 582, 122-130.

Brasil. Agência Nacional de Aviação Civil - ANAC (2014). Regulamento Brasileiro da Aviação Civil RBAC $n^{\circ} 145$ Emenda $n^{\circ}$ 01. Organizações de Manutenção de Produto Aeronáutico. Brasília, DF: ANAC.

Bryman, A., \& Bell, E. (2007). Business research methods. New York: Oxford University Press.

Cheung, A., Ip, W. H., \& Lu, D. (2005). Expert system for aircraft maintenance services industry. Journal of Quality in Maintenance Engineering, 11(4), 348-358. http://dx.doi.org/10.1108/13552510510626972.

Chockie, A., \& Bjorkelo, K. (1992). Effective maintenance practices to manage system aging. In Proceedings of the Annual Reliability and Maintainability Symposium (pp. 166-170). Las Vegas: IEEE. http:// dx.doi.org/10.1109/ARMS.1992.187817.

Cooper, D. R., \& Emory, C. W. (1995). Business research methods. Chicago: McGraw-Hill.

Fleury, A., \& Fleury, M. T. L. (2003). Estratégias competitivas e competências essenciais: perspectivas para a internacionalização da indústria no Brasil. Gestão \& Produção, 10(2), 129-144. http://dx.doi.org/10.1590/ S0104-530X2003000200002.

Fleury, A., \& Fleury, M. T. L. (2004). Estratégias empresariais e formação de competências: um quebra cabeças caleidoscópio da indústria brasileira (3 ed.). São Paulo: Atlas.

Garg, A., \& Deshmukh, S. G. (2006). Maintenance management: literature review and directions. Journal of Quality in Maintenance Engineering, 12(3), 205-238. http://dx.doi.org/10.1108/13552510610685075.

Knotts, R. M. H. (1999). Civil aircraft maintenance and support: fault diagnosis from a business perspective. Journal of Quality in Mainte- 
nance Engineering, 5(4), 335-347. http://dx.doi. org/10.1108/13552519910298091.

Martin, H. H. (1997). Contracting out maintenance and a plan for future research. Journal of Quality in Maintenance Engineering, 3(2), 81-90. http://dx.doi. org/10.1108/13552519710167700.

Mata, J. N., Fo., Miranda, C. F., \& Lima, C. E. (1998). Manutenção baseada em confiabilidade e controle de custos de manutenção - um time de sucesso na indústria aeronáutica. In Anais do XIII Congresso Brasileiro de Manutenção (pp. 1-10). Salvador: Abraman.

Mckinsey \& Company (2010). Estudo do setor de transporte aéreo do Brasil: relatório consolidado. Rio de Janeiro: McKinsey \& Company.

Mishra, R. P., Anand, G., \& Kodali, R. (2006). Development of a framework for world-class maintenance systems. Journal of Advanced Manufacturing Systems, 5(2), 141-165. http://dx.doi.org/10.1142/ S0219686706000807.

Moayed, F. A., \& Shell, R. L. (2009). Comparison and evaluation of maintenance operations in lean versus non-lean production systems. Journal of Quality in Maintenance Engineering, 15(3), 285-296. http:// dx.doi.org/10.1108/13552510910983224.

Nascimento, F. S. (2006). Proposta de modelo de previsão aplicado aos custos de manutenção de aeronaves militares (Dissertação de mestrado). Programa de Engenharia Aeronáutica e Mecânica, Instituto Tecnológico de Aeronáutica, São José dos Campos.

Papakostas, N., Papachatzakis, P., Xanthakis, V., Mourtzis, D., \& Chryssolouris, G. (2010). An approach to operational aircraft maintenance planning. Decision Support Systems, 48(4), 604-612. http://dx.doi. org/10.1016/j.dss.2009.11.010.

Pintelon, L., Pinjala, S. K., \& Vereecke, A. (2006). Evaluating the effectiveness of maintenance strategies. Journal of Quality in Maintenance Engineering, 12(1), 7-20. http://dx.doi.org/10.1108/13552510610654501.

Prahalad, C. K., \& Hamel, G. (1990). The core competence of the corporation. Harvard Business Review, 68(3), 79-91.

Pun, K.-F., Chin, K.-S., Chow, M.-F., \& Lau, H. C. W. (2002). An effectiviness-centred approach to maintenance. a case study. Journal of Quality in Maintenance Engineering, 8(4), 346-368. http://dx.doi. org/10.1108/13552510210448559.

Quivy, R., \& Van Campenhoudt, L. (1998). Manual de investigação em ciências sociais (2 ed.). Lisboa: Gradiva.
Rodrigues, L. R., Gomes, J. P. P., Bizarria, C. O., Galvao, R. K. H., \& Yoneyama, T. (2010). Using prognostic system and decision analysis techniques in aircraft maintenance cost-benefit models. In Proceedings of the Aerospace Conference, 2010 IEEE (pp. 1-7). Big Sky: IEEE. http://dx.doi.org/10.1109/ AERO.2010.5446839.

Samaranayake, P. (2006). Current practices and problem areas in aircraft maintenance planning and scheduling - interfaced/integrated system perspective. In Proceedings of the VII Asia Pacific Industrial Engineering and Management Systems Conference (pp. 2245-2256). Bangkok: Asian Institute of Technology.

Samaranayake, P., Kiridena, S., Laosirihongthong, T., Tsang, A. H. C., \& Xie, M. (2007). Reliability improvements in aircraft maintenance planning and scheduling under uncertainty. In Proceedings of the V International Conference on Quality and Reliability (pp. 49-55). Chiang Mai: Research Publishing Services.

Sherwin, D. (2000). A review of overall models for maintenance management. Journal of Quality in Maintenance Engineering, 6(3), 138-164. http://dx.doi. org/10.1108/13552510010341171.

Silva, R. I., Fo., Aguiar, M. A., \& Kovács, Z. L. (2005). Um estudo de caso genérico de diagnóstico preditivo para sistemas complexos. Anais do IV SITRAER (pp. 1-10). São José dos Campos: Sociedade Brasileira de Transporte Aéreo - SBTA.

Tsang, A. H. C. (1998). A strategic approach to managing maintenance performance. Journal of Quality in Maintenance Engineering, 4(2), 87-94. http://dx.doi. org/10.1108/13552519810213581.

Tu, P. V. L., Yam, R., Tse, P., \& Sun, A. O. (2001). An integrated maintenance management system for an advanced manufacturing company. International Journal of Advanced Manufacturing Technology, 17(9), 692-703. http://dx.doi.org/10.1007/s001700170135.

Vilela, J. A. B. M., Velasquez, E. A. S., Mata, J. N., Fo., \& Kikko, F. (2010). Manutenção em aeronaves: fator contribuinte para a Segurança de Aviação. Revista Conexão SIPAER, 1(2), 85-99.

Voss, C., Tsikriktsis, N., \& Frohlich, M. (2002). Case research in operations management. International Journal of Operations \& Production Management, 22(2), 195-219. http://dx.doi. org/10.1108/01443570210414329.

Waeyenbergh, G., \& Pintelon, L. (2002). A framework for maintenance concept development. International Journal of Production Economics, 77(3), 299-313. http://dx.doi.org/10.1016/S0925-5273(01)00156-6. 